Original Paper

\title{
Analysis of Psychoactive Drugs by Temperature-Responsive Chromatography
}

\author{
Toshiyasu MIKUMAa, Tatsumi KUROKI, Mitsuki YOSHIKAWA, Ryo UCHIDA, Yuki HIRUTA, \\ Hideko KANAZAWA*
}

Faculty of Pharmacy, Keio University, 1-5-30, Shibakoen, Minato-ku, Tokyo 105-8512, Japan

Present address: ${ }^{a}$ Division of Physical and Analytical Chemistry, Nihon Pharmaceutical University, 10281, Komuro, Ina 362-0806, Japan

\begin{abstract}
Temperature-responsive chromatography, in which the characteristics of the stationary phase can be controlled by varying the column temperature with only an aqueous eluent, was applied to the analysis of psychoactive drugs. Temperature-responsive poly( $N$-isopropylacrylamide) (PNIPAAm)-based copolymers were synthesized with $n$-butyl methacrylate (BMA) and $N$-acryloyl L-phenylalanine methyl ester (Phe-OMe) as a comonomer. These polymers were grafted onto aminopropyl silica and used as the stationary phase. Seven psychoactive drugs could be separated simultaneously by the column, including BMA. Among the seven drugs, for triazolam, which has a triazole ring in its structure, the order of elution changed on changing the column temperature. The phenomenon could be explained by $\mathrm{NH}-\pi$ interactions between the $\mathrm{NH}$ groups of PNIPAAm and $\pi$ electrons of the triazole ring. The conformational changes of PNIPAAm altered the degree of exposure of the NH groups and would affect the elution order of the analytes. To enhance the molecular recognition ability for the triazole ring, the column containing Phe-OMe was used. Noticeable changes in the retention factors occurred due to the additional $\pi-\pi$ interactions between the phenyl moieties of Phe-OMe and the triazole ring. The effects of the $\pi-\pi$ interactions were also altered by the changes in column temperature. This chromatographic system, which needs no toxic organic solvent, offers an effective way to determine the causes of addiction in medical institutions.
\end{abstract}

Keywords: Temperature-responsive chromatography; Drug; Molecular recognition

\section{Introduction}

Drug addiction is a worldwide problem. Psychoactive drugs are often abused and because of their toxic symptoms, addicts are frequently admitted to hospitals. In order to determine the appropriate treatment plan for the addicts, medical doctors and their staff have to identify the cause of poisoning quickly. Further, in Japan, the outline of medical fee was revised by the government in 2014 [1], and the number of hospitals that can get a reward for using instrumental analyses to determine the causes of addiction have increased. A continuous effort is needed to develop effective analytical methods which can be used in hospitals.

High-performance liquid chromatography (HPLC) is the

*Corresponding author: Hideko KANAZAWA

Tel.: +81-3-5400-2657; Fax: +81-3-5400-1378

E-mail: kanazawa-hd@pha.keio.ac.jp preferred technique for the analyses of drugs and their metabolites in biological fluids. Various columns with different ways of separating analytes have been reported and are commercially sold. Among them, reversed-phase liquid chromatography (RPLC) is known to be a powerful tool for the determination of causative substances in toxicology. However, the use of RPLC in hospitals has been restricted because RPLC generally requires the use of toxic organic solvents as the mobile phase.

We have been investigating temperature-responsive chromatography, in which a thermo-responsive polymer such as poly $(N$-isopropylacrylamide) (PNIPAAm) is used as the stationary phase [2-16]. PNIPAAm exhibits thermally

Received: 5 July 2017

Accepted: 13 September 2017

J-STAGE Advance Published: 28 September 2017

DOI: $10.15583 /$ jpchrom.2017.011 
<smiles>C=CCC1(CC=C)C(=O)NC(=O)NC1=O</smiles>

Allobarbital

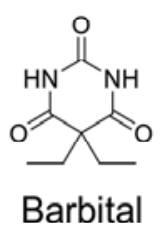

Barbital

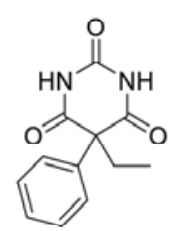

Phenobarbital<smiles>CN1C(=O)CN=C(c2ccccc2)c2cc(Cl)ccc21</smiles>

Diazepam<smiles>O=C1CN=C(c2ccccc2)c2cc([N+](=O)[O-])ccc2N1</smiles>

Nitrazepam<smiles>CN1C(=O)CN=C(c2ccccc2F)c2cc([N+](=O)[O-])ccc21</smiles>

Flunitrazepam<smiles>Cc1nnc2n1-c1ccc(Cl)cc1C(c1ccccc1Cl)=NC2</smiles>

Triazolam<smiles>Clc1ccc2c(c1)C(c1ccccc1)=NCc1nncn1-2</smiles>

Estazolam<smiles>Cc1nnc2n1-c1ccc(Cl)cc1C(c1ccccc1)=NC2</smiles>

Alprazolam<smiles>Cc1nnc2n1-c1sc(Br)cc1C(c1ccccc1Cl)=NC2</smiles>

Brotizolam<smiles>CCc1cc2c(s1)-n1c(C)nnc1CN=C2c1ccccc1Cl</smiles>

Etizolam

Fig. 1. Barbiturates and benzodiazepines used as analytes.

reversible solubility changes in aqueous solutions in response to temperature changes across its lower critical solution temperature (LCST). The polymer chains of PNIPAAm are hydrophilic and hydrated in water below the LCST, whereas above, they are hydrophobic and dehydrated. Consequently, the polymer-grafted stationary phase exhibits temperature-regulated hydrophilic/hydrophobic characteristics as a result of the polymer's conformational changes. In this chromatographic system, the retention times of analytes can be controlled by a change in the column temperature while only using an aqueous mobile phase without any toxic organic solvents.

The characteristics of PNIPAAm can be modified by introducing various comonomers into the polymer unit. For example, using $n$-butyl methacrylate (BMA) as a hydrophobic comonomer leads to increased hydrophobicity [8]. Recently, Hiruta et al. reported that aromatic amino acids like L-phenylalanine and L-tryptophan were introduced to PNIPAAm as a comonomer and a new separation mode featuring molecular recognition was successfully introduced to the stationary phase, due to $\pi-\pi$ interactions and hydrogen bonding [16].

Here, we examined the applicability of temperature-responsive chromatography to the analysis of two groups of psychoactive drugs: barbiturates and benzodiazepines (Fig. 1). The goal of this study was to find basic knowledge for the separations of psychoactive drugs by this chromatographic system aiming the use in medical institutions. Two types of temperature-responsive PNIPAAm-based copolymers were synthesized with BMA and $\mathrm{N}$-acryloyl L-phenylalanine methyl ester (Phe-OMe).
These polymers, P(NIPAAm-co-BMA) and P(NIPAAm-co-Phe-OMe) (Fig. 2), were grafted onto aminopropyl silica and used as the stationary phases. In this study, the unique molecular recognition of some benzodiazepines was observed and the ability of the column to recognize benzodiazepines could be altered by changing the column temperature. The characteristics and molecular recognition abilities of the temperature-responsive chromatography were investigated.

\section{Materials and methods}

\subsection{Reagents and chemicals}

$\mathrm{N}$-Isopropylacrylamide (NIPAAm), kindly provided by KJ Chemicals Co., Ltd. (Tokyo, Japan), was purified by recrystallization from $n$-hexane, and then dried at $25{ }^{\circ} \mathrm{C}$ under vacuum. Phe-OMe was synthesized as previously reported [17,18]. 2,2'-Azobisisobutyronitrile (AIBN) was purchased from Wako Pure Chemical Industries (Osaka, Japan). 3-Mercaptopropionic acid (MPA) and $N, N^{\prime}$-dicyclohexylcarbodiimide (DCC) were purchased from Kanto Chemicals (Tokyo, Japan). $N$-Hydroxysuccinimide (NHS) was purchased from Merck Japan (Tokyo, Japan). Aminopropyl silica (average diameter $=3 \mu \mathrm{m}$ ) was purchased from Nishio Kogyo (Tokyo, Japan). Uracil, barbital, phenobarbital, flunitrazepam, diazepam, brotizolam, and estazolam were purchased from Wako Pure Chemical Industries. Allobarbital and etizolam were purchased from Tokyo Chemical Industry (Tokyo, Japan). Triazolam, nitrazepam, and alprazolam were purchased from Sigma-Aldrich (Louis, MO, USA). Deionized water treated by a Millipore Milli-Q 


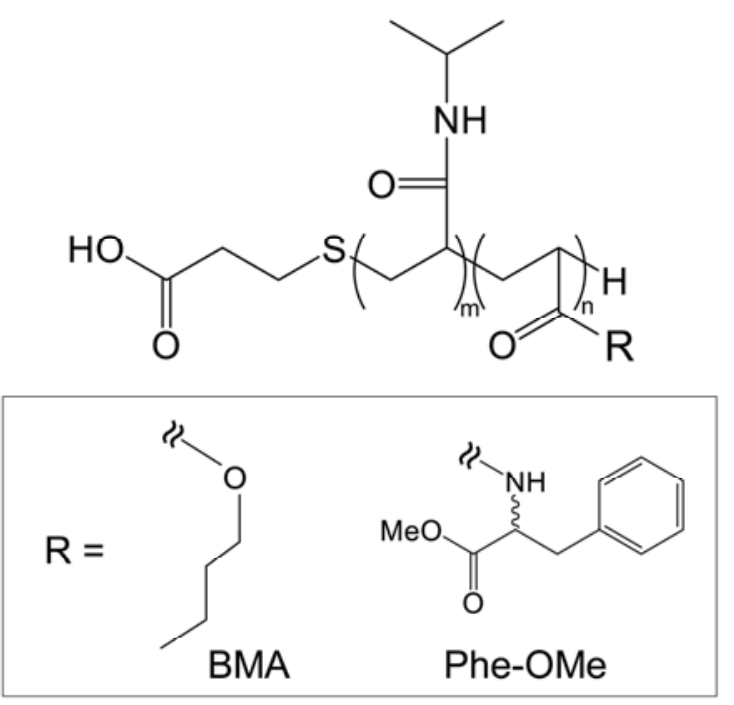

Fig. 2. Structures of PNIPAAm-based temperature-responsive polymers used in this study.

System (Bedford, MA, USA) was used for preparation of the eluent and samples. All other chemicals were of analytical reagent grade.

\subsection{Synthesis of the temperature-responsive polymers}

L-Phenylalanine was converted into the methyl ester using $\mathrm{SOCl}_{2}$ in $\mathrm{MeOH}$ [19]. Phe-OMe was synthesized through a reaction with acryloyl chloride and the methyl ester in the presence of trimethylamine [17,18]. P(NIPAAm-co-BMA) and P(NIPAAm-co-Phe-OMe) were prepared using radical polymerization. NIPAAm (18.0 g, 159 mmol, 95 equiv.) and BMA (1.20 g, $8.40 \mathrm{mmol}, 5$ equiv.) or Phe-OMe (1.96 g, $8.40 \mathrm{mmol}, 5$ equiv.) were dissolved in $N, N$-dimethylformamide (DMF, $45 \mathrm{~mL}$ ). AIBN (0.116 g, $0.706 \mathrm{mmol}, 0.4$ equiv.) and MPA (0.496 g, 4.13 mmol, 2.5 equiv.), which act as the radical initiator and chain transfer agent, respectively, were added to the solution. The reaction mixture was degassed using freeze-pump-thaw cycles and then heated to $70{ }^{\circ} \mathrm{C}$. After reacting for $5 \mathrm{~h}$, the solution was poured into diethyl ether to precipitate the polymer. The crude product was further purified by repeated precipitation from an acetone solution into diethyl ether and was then dried to yield a white solid.

\subsection{Characterization of the temperature-responsive polymers}

The ${ }^{1} \mathrm{H}$ NMR spectra were recorded at $500 \mathrm{MHz}$ on a Varian INOVA-500 spectrometer. The LCSTs of the polymers were determined by measuring their optical transmittance in aqueous solution $(0.5 \%)$. The optical transmittance was measured at various temperatures at 500 $\mathrm{nm}$ using a UV-Vis spectrophotometer (V-630, JASCO,
Tokyo, Japan). The temperature was controlled using an ETC-717 controller (JASCO) and a PT-31 Peltier system (Krüss, Hamburg, Germany) at a heating rate of $0.1{ }^{\circ} \mathrm{C}$ $\min ^{-1}$. The LCSTs were determined to be the temperature at which the polymer solutions had $50 \%$ optical transmittance.

\subsection{Modification of aminopropyl silica with the temperature-responsive polymers \\ P(NIPAAm-co-BMA) (1.5 g) or}

$\mathrm{P}(\mathrm{NIPAAm}-\mathrm{co}-\mathrm{Phe}-\mathrm{OMe})(1.5 \mathrm{~g})$ was dissolved in $20 \mathrm{~mL}$ of ethyl acetate. DCC $(0.16 \mathrm{~g})$ and NHS $(0.09 \mathrm{~g})$ were then added to the solution. The reaction mixture was stirred at $4{ }^{\circ} \mathrm{C}$ for $2 \mathrm{~h}$ and then at $25^{\circ} \mathrm{C}$ for an additional $24 \mathrm{~h}$. The precipitated dicyclohexylurea was removed by filtration, and the filtrate was poured into diethyl ether to precipitate the polymer. The resulting residue with activated terminal carboxylic acid $(0.5 \mathrm{~g})$ was dissolved in $25 \mathrm{~mL}$ of 1,4-dioxane. To this was added $1.0 \mathrm{~g}$ of aminopropyl silica. The reaction mixture was shaken at $25^{\circ} \mathrm{C}$ for $24 \mathrm{~h}$. This process was repeated three times. The polymer-modified silica was washed with $100 \mathrm{~mL}$ of ethyl acetate and dried under vacuum at ambient temperature. After drying, the silica was dissolved in $20 \mathrm{~mL}$ of 1,4-dioxane with glycidol $(10 \mathrm{~mL})$. The mixture was shaken at $25{ }^{\circ} \mathrm{C}$ for $24 \mathrm{~h}$ for end-capping of the silica's amino group. The resulting silica was washed with $100 \mathrm{~mL}$ of ethyl acetate and dried under vacuum at ambient temperature.

\subsection{Temperature-responsive chromatography}

The polymer-grafted silica beads were packed into a stainless-steel column $(100 \mathrm{~mm}$ length $\times 2.1 \mathrm{~mm}$ i.d. $)$. The column was then connected to an HPLC system. HPLC analysis was performed using a Prominence- $i$ LC2030C (Shimadzu, Kyoto, Japan). The detection wavelength was $220 \mathrm{~nm}$. The eluent was $10 \mathrm{mM}$ ammonium acetate $(\mathrm{pH}$ 4.8 ) at a flow rate of $0.2 \mathrm{~mL} \mathrm{~min}^{-1}$. All drugs were dissolved in tetrahydrofuran at a concentration of $1.0 \mathrm{mg} \mathrm{mL}^{-1}$ for use as stock solutions, and all drug samples were prepared at a concentration of $20 \mu \mathrm{g} \mathrm{mL}^{-1}$ by diluting the stock solutions with deionized water. The retention factors of the analytes were calculated using the following equation: retention factor $=\left(t_{\mathrm{R}}-t_{0}\right) / t_{0}$, where $t_{0}$ and $t_{\mathrm{R}}$ are the retention times of uracil and the target analyte, respectively.

\section{6. $R P L C$}

In the RPLC analysis for the comparison with temperature-responsive chromatography, COSMOSIL $5 \mathrm{C}_{18}$-AR-II column $(150 \mathrm{~mm}$ length $\times 4.6 \mathrm{~mm}$ i.d., $5 \mu \mathrm{m})$ (Nacalai, Kyoto, Japan) was used. The eluent was $10 \mathrm{mM}$ ammonium acetate ( $\mathrm{pH} 4.8)$ /methanol (50:50) at a flow rate of $1.0 \mathrm{~mL} \mathrm{~min}^{-1}$. The other conditions were same with those in section 2.5 . 


\section{Results and discussion}

\subsection{Temperature response of PNIPAAm copolymers}

$\mathrm{P}(\mathrm{NIPAAm}-\mathrm{co}-\mathrm{BMA})$ and P(NIPAAm-co-Phe-OMe) were synthesized from NIPAAm with BMA and Phe-OMe, respectively, through radical polymerization using MPA. Although the feed contents of the comonomers in the polymerization reaction were set at $5 \%$, the actual introduction rates were determined by ${ }^{1} \mathrm{H}$ NMR analysis (data not shown). The NIPAAm/comonomer ratios of the polymers were estimated using the integrated signals derived from the NIPAAm methine $(1 \mathrm{H}, 3.9 \mathrm{ppm})$ proton, BMA methyl (3H, $0.9 \mathrm{ppm})$ proton, and the aromatic ring of the Phe-OMe (5H, 7.0-7.3 ppm). The monomer composition of the polymers was found to be similar to their initial feed composition. Table 1 summarizes the polymer characterization data. The molecular weights and LCSTs determined in this study corresponded well with those reported previously [16]. These results indicated that the objective polymers were successfully synthesized.

\subsection{The characteristics of temperature-responsive} chromatography

Seven types of psychoactive drugs were analyzed using the P(NIPAAm-co-BMA) column at various column temperatures (Fig. 3). Seven drugs showed good separations only with aqueous mobile phase. When the column temperature was increased, the retention times of the drugs were retarded overall. By contrast, the opposite behavior was observed in the conventional RPLC system. The analytes retention decreased on increasing the column temperature. This phenomenon could be explained by the thermally dependent conformational changes of the polymer, because of which interactions between the analytes and stationary phase became stronger as the column temperature was increased and the surface property of the stationary phase changed from hydrophilic to hydrophobic. Fig. 4(a)

Table 1. Physicochemical properties of the PNIPAAm-based polymers

\begin{tabular}{cccc}
\hline Polymers & $\begin{array}{c}\text { Composition ratios } \\
\text { of a comonomer }\end{array}$ & $M^{\mathrm{b}}$ & LCST $\left({ }^{\circ} \mathrm{C}\right)$ \\
\hline $\begin{array}{c}\text { P(NIPAAm- } \\
\text { co-BMA) }\end{array}$ & $4.8 \%$ & 6100 & 24.2 \\
$\begin{array}{c}\text { P(NIPAAm- } \\
\text { co-Phe-OMe) }\end{array}$ & $4.9 \%$ & 6500 & 23.7 \\
\hline
\end{tabular}

a. Determined by ${ }^{1} \mathrm{H}$ NMR analyses.

b. Number-averaged molecular weight determined by end group titration.

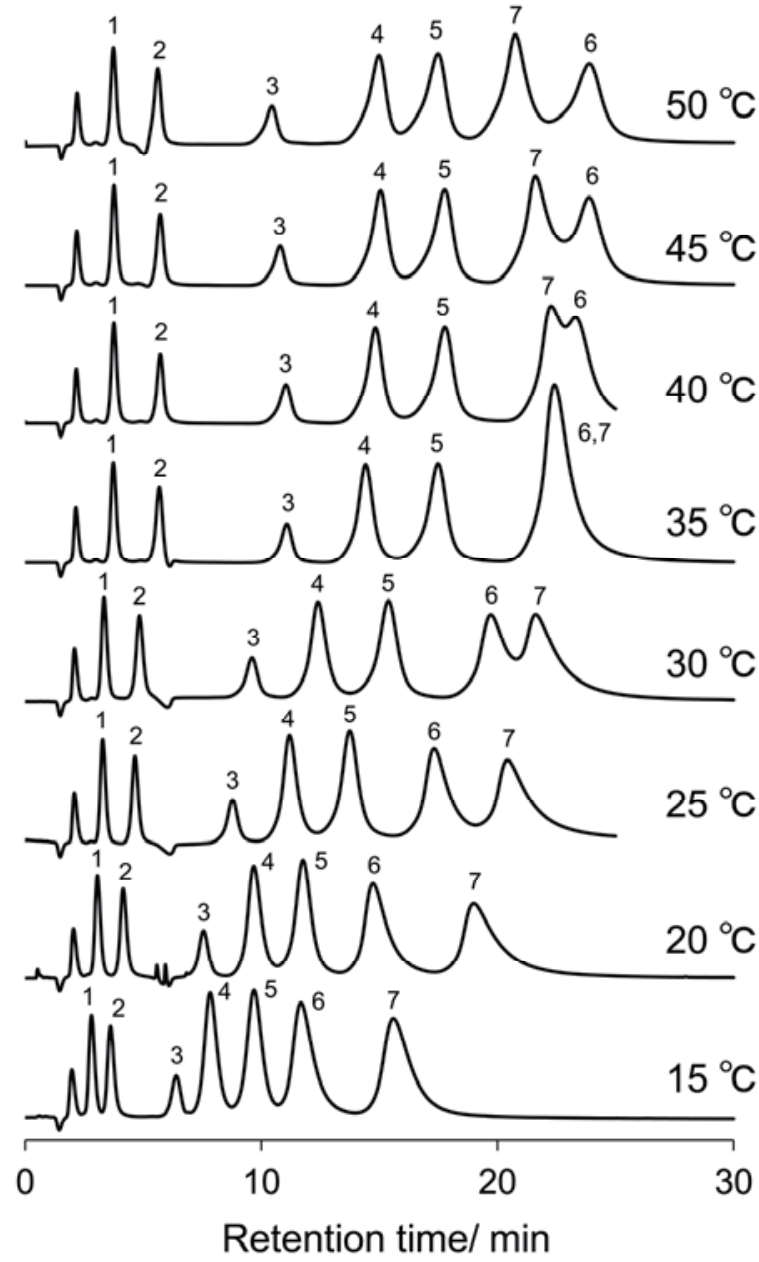

Fig. 3. Chromatograms of the psychoactive drugs obtained from analyses on the P(NIPAAm-co-BMA) column at various temperatures. 1, barbital, 2, allobarbital, 3, phenobaribital, 4, flunitrazepam, 5, nitrazepam, 6, diazepam, 7, triazolam.

shows the van't Hoff plots for the seven drugs on the P(NIPAAm-co-BMA) column. In order to compare these with a conventional technique, the plots obtained from RPLC analysis are also shown in Fig. 4(b). Linearity is clearly observed in the RPLC system. However, in the P(NIPAAm-co-BMA) column, a deviation from linearity was found between the $\ln k$ values and $1 / T$. Interestingly, the slope of the van't Hoff plots of each analyte on the P(NIPAAm-co-BMA) column changed markedly at the LCST boundary. This corresponds to a phase transition of the polymer at the surface of the stationary phase. Additionally, the slope of the van't Hoff plots for the $\mathrm{P}$ (NIPAAm-co-BMA) column is negative, in contrast to the positive slopes observed for conventional RPLC. This provided additional evidence that the interaction between the drugs and temperature-responsive surface became stronger at elevated column temperatures. 
(a)

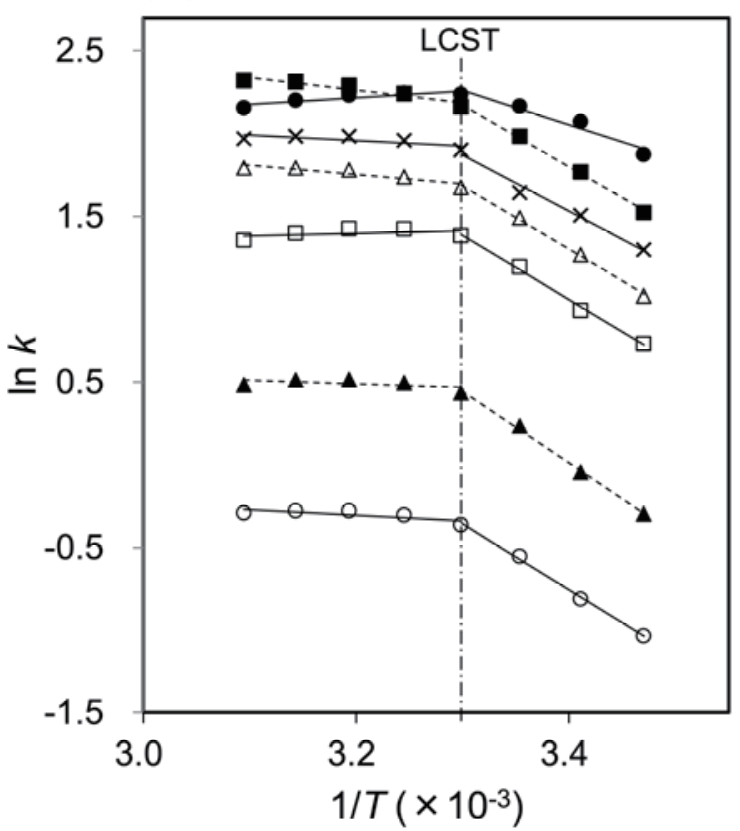

(b)

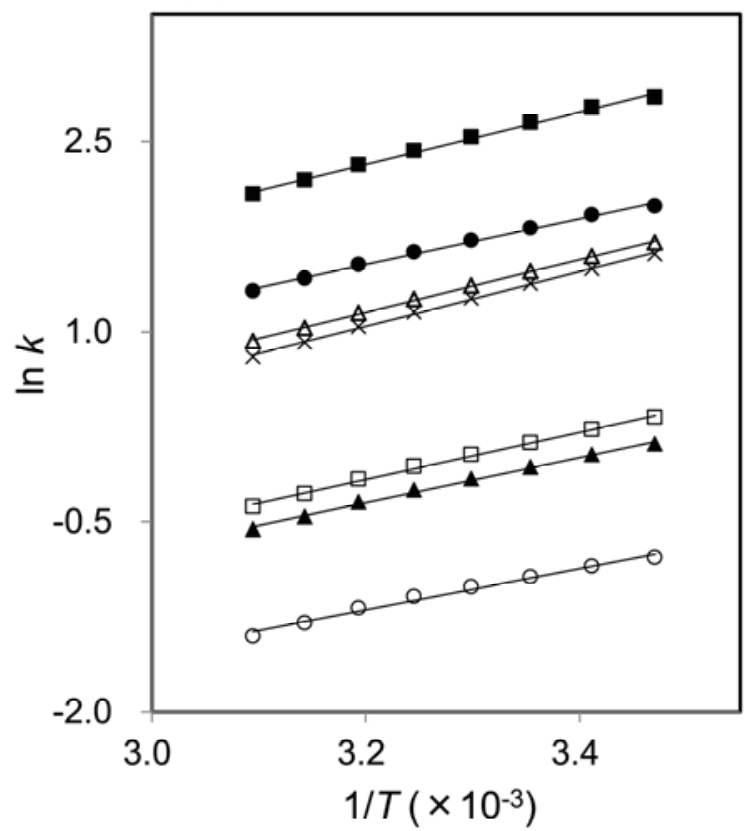

Fig. 4. van't Hoff plots of the psychoactive drugs (a) on the P(NIPAAm-co-BMA) column and (b) from the conventional RPLC system. $\bigcirc$, barbital, $\boldsymbol{\Lambda}$, allobarbital, $\square$, phenobaribital, $\triangle$, flunitrazepam, $\times$, nitrazepam, $\mathbf{\square}$, diazepam, $\boldsymbol{\odot}$, triazolam.

\subsection{Molecular recognition in the P(NIPAAm-co-BMA) column}

Interestingly, in Fig. 3, the order of elution of diazepam and triazolam was changed at column temperatures of $30-40{ }^{\circ} \mathrm{C}$. Fig. 5 shows the plots of the retention factor against the column temperature obtained from Fig. 3. It could be interpreted that the retention factors of triazolam at lower column temperatures were markedly higher compared to those of the other drugs. In the comparison of the chemical structures of the seven drugs, only triazolam has a triazole ring in its structure. To confirm the effect of the triazole ring on the retention factors, five triazolobenzodiazepines, which contain a triazole ring in their structures, were analyzed alongside some benzodiazepines that had no triazole ring. The retention factors obtained at each column temperature were normalized at $50{ }^{\circ} \mathrm{C}$, and the plots of the relative retention factors against column temperature are shown in Fig. 6(a). Based on the retention factors against the column temperatures, the compounds were classified into two types: triazolobenzodiazepines and other benzodiazepines. These results clearly indicated that the triazole ring was the cause of the increase in retention factors at lower column temperatures. The molecular interactions driving the above phenomenon could be explained by the increased $\mathrm{NH}-\pi$ interactions between $\mathrm{NH}$ groups of the PNIPAAm and $\pi$ electrons of the triazole ring (Fig. 7). At lower temperatures, the NH groups of PNIPAAm were exposed and the polymer chains were hydrated. Therefore, the analytes could easily interact with the $\mathrm{NH}$ groups through $\mathrm{NH}-\pi$ interactions. On the other hand, at higher temperatures, the $\mathrm{NH}-\pi$

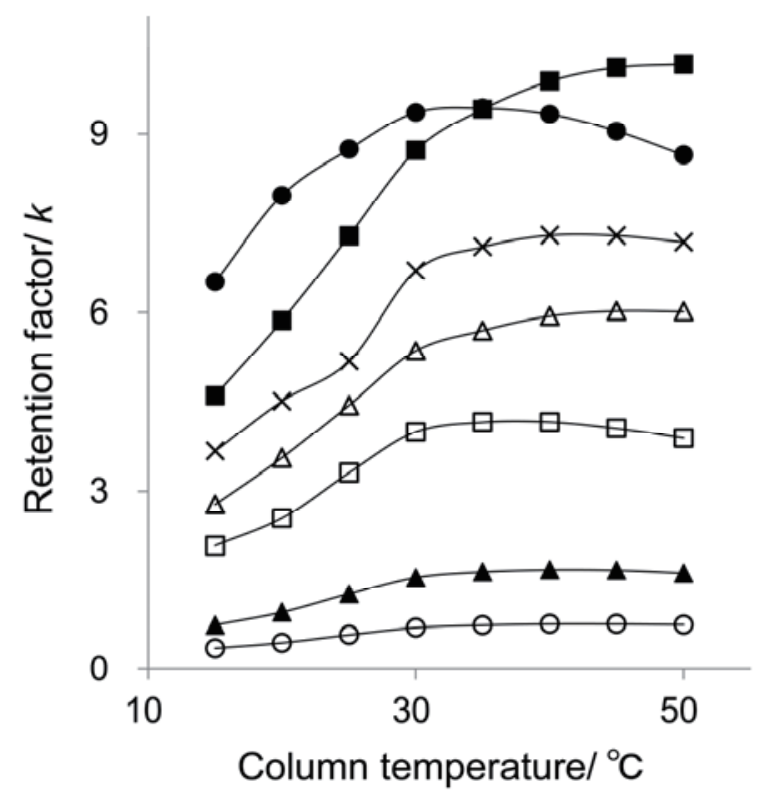

Fig. 5. Plots of the retention factors of the psychoactive drugs against column temperatures on the P(NIPAAm-co-BMA) column. $\bigcirc$, barbital, $\boldsymbol{\Delta}$, allobarbital, $\square$, phenobaribital, $\triangle$, flunitrazepam, $\times$, nitrazepam, $\mathbf{\square}$, diazepam, $\boldsymbol{O}$, triazolam. 
(a)

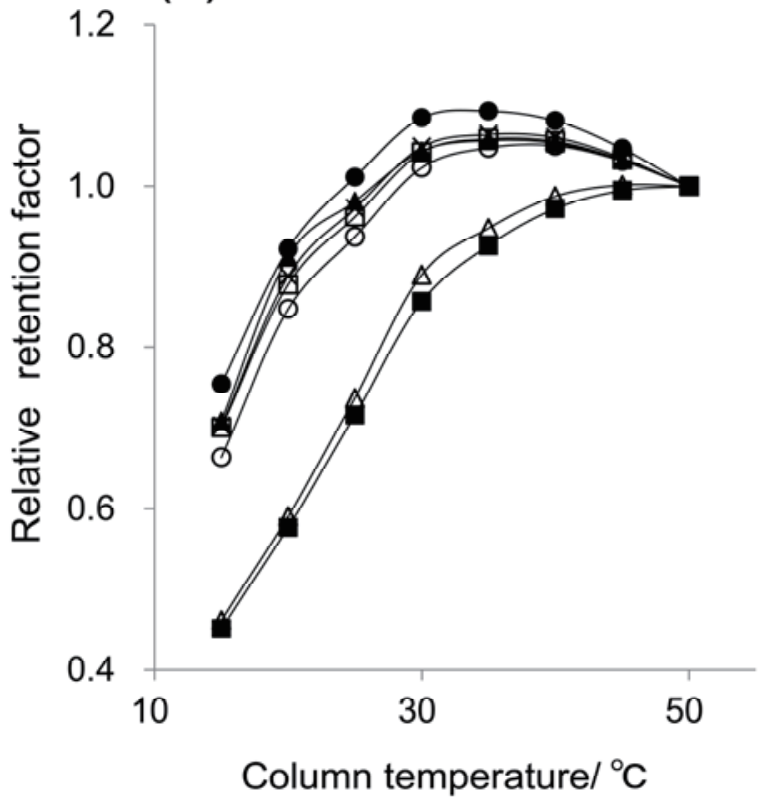

(b)

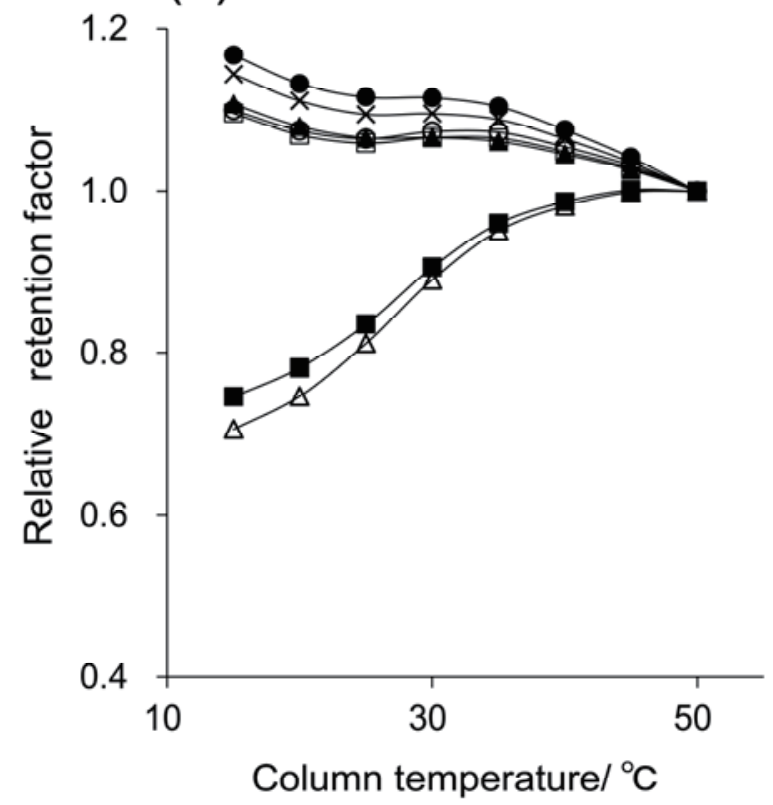

Fig. 6. Plots of the relative retention factors of the benzodiazepine analytes against the column temperatures on (a) P(NIPAAm-co-BMA) column and (b) P(NIPAAm-co-Phe-OMe) column. The normalizations were carried out at $50{ }^{\circ} \mathrm{C}$. $\bigcirc$, etizolam, $\boldsymbol{\Delta}$, alprazolam, $\square$, estazolam, $\triangle$, flunitrazepam, $\times$, brotizolam, $\mathbf{\square}$, diazepam, $\bigcirc$, triazolam.

interactions were weakened because the NH groups were hidden in the shrunken dehydrated polymer chains. Since all benzodiazepines have two benzene rings in their structures, interaction between the NH groups and the $\pi$ electrons of the benzene rings may have occurred for all benzodiazepines. However, when the differences in the retention factors are discussed, the affinities derived from these benzene rings can be ignored because the contributions from the benzenes may be negligible. Further, it is known that the triazole ring has an excess of $\pi$ electrons ( $\operatorname{six} \pi$ electrons on five atoms), and that the electron density of triazole is higher than that of the benzene ring [20]. Therefore, the interactions between the $\mathrm{NH}$ groups of the stationary phase and the triazole ring would be stronger than the NH- $\pi$ interactions with the benzene ring, leading to a notable impact on the retention factors of triazolobenzodiazepines.

\subsection{Molecular recognition in the P(NIPAAm-co-Phe-OMe) column}

To enhance the molecular recognition of the triazole ring, the P(NIPAAm-co-Phe-OMe) column, which has phenyl moieties as the molecular recognition sites, was used for the analysis of the same analytes in Fig. 6(a). As with the NH groups of PNIPAAm, the phenyl moieties were widely distributed in the polymer. Therefore, it was expected that the effects derived from the phenyl moieties (i.e. $\pi-\pi$ interactions) would be observed particularly at low column temperatures because of the polymer extension. The retention factors obtained at each column temperature were normalized at $50{ }^{\circ} \mathrm{C}$, and the plots of the relative retention factors against the column temperature are shown in Fig. 6(b). At lower temperatures, the relative retention factors of all analytes were higher than those in the P(NIPAAm-co-BMA) column. These results suggested that $\pi-\pi$ interactions were occurring not only with the triazole ring but also with the benzene rings, causing a general increase in the retention factors. As is the case with the P(NIPAAm-co-BMA) column, the changes of the retention factors with column temperature could be classified into two types: triazolobenzodiazepines and other benzodiazepines. Further, the increases of the relative retention factors for triazolobenzodiazepines at lower column temperatures were conspicuous. These results indicated that the interactions between the triazole ring and the phenyl moieties contributed to the enhancement of column affinity through $\pi-\pi$ interactions. Recall that the P(NIPAAm-co-Phe-OMe) column had only $5 \%$ Phe-OMe as a comonomer. Therefore, it followed that inclusion of only $5 \%$ comonomer led to notable differences in the retention factors. The phenyl moieties of Phe-OMe could have great potential for use as molecular recognition sites of the triazole ring. 


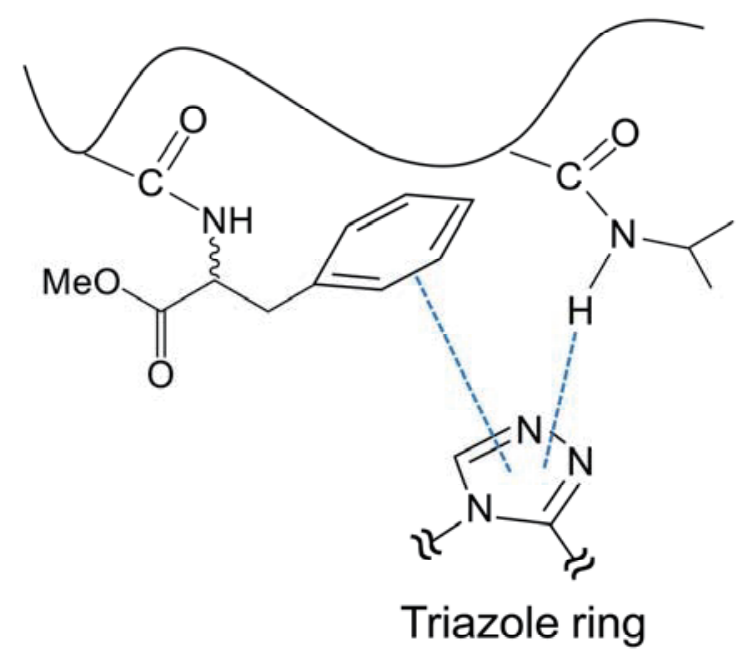

Fig. 7. Schematic representation of the interactions with the triazole ring. (right side) The $\mathrm{NH}-\pi$ interactions with $\mathrm{NH}$ groups of the PNIPAAm, (left side) the $\pi-\pi$ interactions with phenyl moieties of the Phe-OMe.

\section{Conclusions}

Seven psychoactive drugs were analyzed by temperature-responsive chromatography. These drugs showed good separation using the P(NIPAAm-co-BMA) column with only an aqueous mobile phase, and their retention factors could be altered by changing the column temperature. The order of elution for diazepam and triazolam inverted when the column temperature changed from 30 to $40{ }^{\circ} \mathrm{C}$. This phenomenon could be explained by the $\mathrm{NH}-\pi$ interactions occurring between $\mathrm{NH}$ groups of the PNIPAAm stationary phase and the $\pi$ electrons of the triazole ring. To enhance the molecular recognition of the triazole ring, the $\mathrm{P}(\mathrm{NIPAAm}-\mathrm{co}-\mathrm{Phe}-\mathrm{OMe})$ column was used. Molecular recognition by the phenyl moieties of P(NIPAAm-co-Phe-OMe) was clearly observed, and the retention factors of benzodiazepines containing the triazole ring were strongly affected by the $\pi-\pi$ interactions.

In this study, specific interactions between some benzodiazepines and the stationary phase of thermo-responsive columns were observed. Various other interactions may be created by varying the comonomers into the polymer unit. This chromatographic system, which needs no toxic organic solvent, could be an effective way to determine the causes of addiction in medical institutions.

\section{Acknowledgements}

This study was supported in part by a Grant-in-Aid for Scientific Research (B) (Grant No. 21390012 and 25293009) from the Ministry of Education, Culture, Sports, Science and Technology, Japan.

\section{References}

[1] Outline of FY 2014 Revision of Medical Fee, Ministry of Health, Labour and Welfare, Japan.

[2] Kanazawa, H.; Yamamoto, K.; Matsushima, Y.; Takai, N.; Kikuchi, A.; Sakurai, Y.; Okano, T. Anal. Chem. 1996, 68, 100-105.

[3] Kanazawa, H.; Sunamoto, T.; Matsushima, Y.; Kikuchi, A.; Okano, T. Anal. Chem. 2000, 72, 5961-5966.

[4] Kanazawa, H.; Sunamoto, T.; Ayano, E.; Matsushima, Y.; Kikuchi, A.; Okano T. Anal. Sci. 2002, 18, 45-48.

[5] Sakamoto, C.; Okada, Y.; Kanazawa, H.; Ayano, E.; Nishimura, T.; Ando, M.; Kikuchi, A.; Okano T. $J$. Chromatogr. A 2004, 1030, 247-253.

[6] Kanazawa, H.; Ayano, E.; Sakamoto, C.; Yoda, R.; Kikuchi, A.; Okano T. J. Chromatogr. A 2006, 1106, 152-158.

[7] Ayano, E.; Okada, Y.; Sakamoto, C.; Kanazawa, H.; Kikuchi, A.; Okano, T. J. Chromatogr. A 2006, 1119, 51-57.

[8] Ayano, E.; Kanazawa, H. J. Sep. Sci. 2006, 29, 738-749.

[9] Kanazawa, H.; Nishikawa, M.; Mizutani, A.; Sakamoto, C.; Morita-Murase, Y.; Nagata, Y.; Kikuchi, A.; Okano, T. J. Chromatogr. A 2008, 1191, 157-161.

[10] Nishio, T.; Suzuki, R.; Tsukada, Y.; Kanazawa, H.; Okano, T.; Miyabe-Nishiwaki, T. J. Chromatogr. A 2009, 1216, 7427-7432.

[11] Nishio, T.; Ayano, E.; Suzuki, Y.; Kanazawa, H.; Okano, T. J. Chromatogr. A 2011, 1218, 2079-2084.

[12] Kanazawa, H.; Okano, T. J. Chromatogr. A 2011, 1218, 8738-8747.

[13] Nishio, T.; Kanazashi, R.; Nojima, A.; Kanazawa, H.; Okano, T. J. Chromatogr. A 2012, 1228, 148-154.

[14] Sakata, K.; Ohkubo, K.; Hiruta, Y.; Ayano, E.; Kanazawa, H. Koubunshi Ronbunsyu 2014, 71, 293-301.

[15] Hiruta, Y.; Nagumo, Y.; Miki, A.; Okano, T; Kanazawa, H. RSC Advances 2015, 5, 73217-73224.

[16] Hiruta, Y.; Kanazashi, R.; Ayano, E.; Okano, T.; Kanazawa, H. Analyst 2016, 141, 910-917.

[17] Sanda, F.; Abe, T.; Endo, T. J. Polym. Sci. Part A: Polym. Chem. 1997, 35, 2619-2629.

[18] Moore, B. L.; O’Reilly, R. K. J. Polym. Sci. Part A: Polym. Chem. 2012, 50, 3567-3574.

[19] Li, K.; Tan, G.; Huang, J.; Song, F.; You, J. Angew. Chem. Int. Ed. 2013, 52, 12942-12945.

[20] Contemporary heterocyclic Chemistry; Newkome, G. R.; Paudler, W. W.; John Wiley \& Sons: USA, 1982. 